\title{
De la ética empresarial a la sostenibilidad, ¿por qué debe interesar a las empresas?
}

\section{From Business Ethics to Sustainability. Why should companies care?}

Itzel

Orozco Martínez

Consultora

independiente

Recibido: 4 de febrero de 2020.

Aprobado: 12 de mayo de 2020. 


\section{Resumen}

Este artículo analiza el contexto histórico que dio origen al concepto de ética empresarial, la teoría de responsabilidad social empresarial y algunos marcos de referencia para la sostenibilidad. Asimismo, considera las aportaciones de cada concepto y analiza por qué la ética empresarial es la base para crear empresas socialmente responsables. Adicionalmente, el artículo plantea cómo las tres variables conviven y se interconectan para crear organizaciones más sostenibles que contribuyan al desarrollo sostenible y a fomentar comunidades más justas y balanceadas. Finalmente, ofrece recomendaciones.

Palabras clave: ética, ética empresarial, responsabilidad social, sostenibilidad, desarrollo sostenible.

Clasificación JEL: M14, Q01, Q56

\section{Abstract}

The article analyzes the origin of business ethics as the root element for corporate social responsibility theory and some sustainability frameworks, while at the same time considering the contribution of each of the three concepts. It also analyzes why business ethics is the main pillar for creating socially responsible organizations and how the three variables coexist and interconnect in order to create more sustainable organizations that contribute to both sustainable development, and more balanced and just communities. Finally, it offers recommendations.

Key Words: ethics, business ethics, social responsibility, sustainability, sustainable development. JEL Classification: M14, Q01, Q56 


\section{Introducción}

Una breve vista al pasado nos deja ver que algunos acontecimientos globales han dejado una huella indeleble en la memoria de la humanidad, tales como las dos guerras mundiales, bombas nucleares, múltiples decisiones gubernamentales y empresariales que han representado escollos en el progreso de la humanidad, vulnerado derechos humanos e incluso cobrado la vida de millones de personas, especies y ecosistemas. A pesar de que tales eventos marcaron un impase que haría difícil ver un futuro mejor, también dieron pauta a la reflexión y elaboración de leyes, decretos, convenciones, normas o estándares que buscan, tanto en el contexto local como en el global, crear un mundo más ético, justo y sostenible.

Todavía falta mucho por hacer, pero merece la pena reconocer los avances y también mencionar que todos los sectores han contribuido. Cabe notar que las empresas, por su actividad productiva, son un actor importante en el desarrollo de las personas y de los países, pues son fuentes de capacitación, desarrollo profesional e incluso personal, generan una importante derrama económica, innovación y, en general, aportan muchas formas de progreso para la sociedad. Por lo tanto, se convierten de facto en ejemplos de conducta, en modelos a seguir. Las empresas son parte de la sociedad, con recursos naturales y humanos llevan a cabo su función productiva y crean bienes y servicios que, en teoría, buscan beneficiar y no perjudicar a la sociedad en general. Por estas razones, las empresas deberían ser responsables de sus acciones y procesos ante la sociedad, así como de la gestión de estos, como lo define la norma ISO 26000 Responsabilidad Social (2010). Es decir, deben ser socialmente responsables o lo que se ha llamado «responsabilidad social empresarial» (RSE). Pero antes que una empresa se proclame socialmente responsable o busque una insignia que avale que lo es, la empresa debe conducirse de forma ética, de lo contrario, corre el riesgo de caer en el «marketing social corporativo» (Licandro, 2016: 25) ० «greenwashing»1 (Delmas y Cuerel, 2011: 65) y perder credibilidad y legitimidad.

La ética empresarial y la filantropía han evolucionado al igual que los temas sociales y ambientales. Por ende, tanto la ética, como las problemáticas ambientales y sociales asumen un rol cada vez más protagónico en la agenda de la alta dirección de empresa y de la política internacional, si no desde un espíritu ético y responsable en 1 Término en inglés que se utiliza para referirse a publicidad engañosa que declara tener beneficios
ambientales o sociales cuando, en realidad, no es cierto. 
origen, al menos por cumplimiento de normas, regulaciones y legislaciones correspondientes, por prevención de riesgos, o por el interés de salvaguardar inversiones y rentabilidad. Por esta razón, en las últimas décadas, se han desarrollado conceptos y sistemas de gestión de RSE, o responsabilidad social corporativa (RSC), como lo Ilaman algunos autores, y de sostenibilidad. Y este último concepto se considera más integral, pues pone en perspectiva a la sociedad y a sus empresas, y las considera parte de un sistema más grande del cual son dependientes: la Tierra o biósfera, como la llama Robèrt (2017).

La ética empresarial es la base para que una empresa sea socialmente responsable y para que contribuya al desarrollo sostenible de la sociedad en la que está inmersa y de la que, a su vez, depende. Entonces surge el cuestionamiento de si la empresa se conduce con ética y toma en cuenta a sus grupos de interés, o stakeholders, en sus procesos y los impactos que estos provocan. Ética empresarial, responsabilidad social empresarial y sostenibilidad son conceptos compatibles y complementarios y, sobre todo, tienen gran relevancia para las empresas en la actualidad, particularmente para aquellas que genuinamente se preocupan por integrar estos temas en su operación.

Este artículo reconoce la aportación de las herramientas de RSE y los modelos de sostenibilidad, identifica que la ética es lo que constituye la base para la empresa que genuinamente se preocupa por integrar estos temas en su operación, y propone algunas recomendaciones.

Las empresas forman parte importante de la actividad económica y productiva de un país, por lo tanto, tienen una posición privilegiada: un gran poder de influencia para encaminar esfuerzos y recursos hacia la sostenibilidad. Sin embargo, en el mundo empresarial se encuentra una brecha entre lo que realmente sucede y la oportunidad de ejercer un liderazgo determinado y más audaz que, ejecutado con altos estándares de ética, coadyuvaría a atender las crisis ambiental y social que vivimos actualmente para hacerlo de forma pronta y oportuna. Como dicen Broman y Robèrt (2005): «La pregunta de si alcanzaremos la sostenibilidad no es si tendremos suficiente energía, comida, u otros recursos. La pregunta es: ¿habrá suficientes líderes a tiempo?».

\section{Metodología}

Para la elaboración del presente trabajo se consultaron diversas fuentes literarias primarias y secundarias, incluidas en la bibliografía. Se revisaron bases de datos de investigación y bibliotecas digitales de revistas académicas tales como 
JSTOR, EBSCOhost, Science Direct, Scopus, Springer por considerarse fuentes documentales serias y respetadas a nivel internacional para investigaciones científicas y académicas.

Por lo anteriormente señalado, el presente trabajo incluye el método documental exploratorio, empírico, empírico-analítico y de investigación documental cualitativa, así como en la experiencia profesional de la autora, que lleva más de quince años trabajando como consultora y catedrática en sostenibilidad, ética empresarial y responsabilidad social.

Con base en la experiencia práctica de la autora del presente trabajo en la atención a diversos clientes corporativos y a partir de los métodos referidos, se investigó y analizó el contexto histórico y la evolución de las prácticas empresariales en torno a las variables antes mencionadas. También se analizó literatura académica relevante para comprender cómo influyó el contexto histórico en el surgimiento de los conceptos mencionados, cómo se entrelazan y cuáles son las herramientas que tienen mayor eficacia e impacto en la gestión de empresa.

\section{Marco conceptual}

En la revisión de la literatura académica, se encuentra que la ética en el mundo de los negocios es una composición heterogénea que se alimenta de conceptos desarrollados por varias disciplinas: filosofía, sociología, política y economía. La ética empresarial no es un tema nuevo, ha ido evolucionando desde los años veinte del siglo XX y, en este tiempo, nacieron el concepto de «responsabilidad social empresarial», el concepto de «stakeholder» y más recientemente el de «sostenibilidad». A continuación, se planeta un contexto histórico, no detallado ni exhaustivo, pero sí enfocado en resaltar algunos hechos y teorías que dieron forma a estos tres conceptos y los han ayudado a evolucionar en una relación simbiótica con el fin de lograr el bienestar de la sociedad y del planeta Tierra.

1930. A finales de los veinte y en los años treinta del siglo XX, la problemática de salarios bajos y condiciones laborales inapropiadas tomó importancia debido a la inconformidad que externaron los trabajadores en protestas. Esto desembocó en programas (aunque controvertidos para algunos economistas) como The New Deal, encaminado a fijar salarios mínimos (Kaufman, 2012), que instauró Franklin D. Roosevelt, entonces presidente de Estados Unidos. Inicialmente, elevar los estándares 
éticos de operación no fue una motivación intrínseca de los empresarios, sino una respuesta motivada por las injusticias laborales e iniciada por los mismos trabajadores, uno de los stakeholders más importantes para las empresas, pues sin ellos no se tiene el capital humano para operar.

1940. Con las secuelas económicas y sociales que dejó la Gran Depresión de los años treinta y la coyuntura de la Segunda Guerra Mundial en los años cuarenta, la preocupación global giraba en torno de la paz y la seguridad. Por ello, con 50 países firmantes de la Carta de las Naciones Unidas, nace en 1945 la Organización de las Naciones Unidas, ONU (1945), organismo supranacional que, desde entonces, dio lugar a numerosos acuerdos aplicables a todos los sectores de todos los países miembros ${ }^{2}$ sobre temas como derechos humanos, justicia social, desarrollo sostenible y, más recientemente, cambio climático, entre muchos más. Tales acuerdos y directrices de organismos multilaterales han influido en el desarrollo teórico de los temas que abordan y viceversa, e inevitablemente han tomado un papel preponderante en la actual agenda empresarial.

1950. En los años cincuenta, el activista Martin Luther King Jr. señaló la necesidad de una mayor justicia social y equidad, dando así inicio y encabezando el movimiento de derechos civiles. Este movimiento subrayó el tema de la discriminación y lo trajo al frente de la agenda política y empresarial de la época, específicamente en Estados Unidos, pero con impacto a escala internacional. Estos acontecimientos, junto con lo que hasta ese momento había evolucionado la ética empresarial, enmarcaron el nacimiento del término «responsabilidad social empresarial» (RSE), y en esta década surgió por primera vez el concepto y se reconoció a Howard Bowen (1953) como el primer proponente de la RSE con su libro Social Responsibilities of Businessmen, aunque su enfoque no era en la empresa, sino en el empresario.

1960. Más adelante, los años sesenta dieron origen al movimiento de la «ecología profunda». Aparecieron las primeras publicaciones y declaraciones de la comunidad científica expresando preocupación sobre el deterioro ambiental y la pérdida de la biodiversidad. La bióloga Rachel Carson publica su famoso libro Silent Spring (1962), en donde hace una fuerte llamada de atención con respecto al uso de pesticidas con

2 Actualmente, 193 Estados soberanos son miembros de la ONU. 
DDT ${ }^{3}$ y su fatídico efecto en el medio ambiente. Su libro es también una profunda reflexión respecto a la interconexión de todos los seres y sistemas vivos, dando pie al nacimiento del pensamiento sistémico.

También en esta década, el científico Clair Patterson (Dickie, 1995) fue pieza fundamental para llevar a la corte de Estados Unidos el asunto del deterioro en la salud del ser humano, y de la naturaleza en general, debido a la gasolina con plomo y aditivos, con lo que expuso a las compañías petroleras que conocían los peligros del uso del plomo, sin embargo, habían hecho caso omiso para no comprometer su planeación de ventas y crecimiento.

1970. En los años setenta apareció por primera vez el término «gobierno corporativo» en el equivalente al Diario Oficial de la Federación de Estados Unidos (Federal Register, 1977). Este concepto busca el balance, la estabilidad y la transparencia en el mundo corporativo. El gobierno corporativo de una empresa es muy importante pues, por medio de este, se establece la estructura de las jerarquías que dirigirá la empresa, los roles y responsabilidades y los códigos de ética o conducta que regirán a la organización.

En la misma década surge la pirámide de RSE de Carroll (1979) en la que propone que las empresas deben basar su actividad en cuatro factores: económico, legal, ético y filantrópico. En la década de 1970, con la pirámide de Carroll puede identificarse cómo la ética empresarial empieza a formar parte integral de la RSE.

1980. En los años ochenta, tras las observaciones y publicaciones de las décadas de 1960 y 1970, la atención hacia temas ambientales comienza a crecer. Se nota un ligero aumento en la sensibilidad sobre estos, debido a que comienzan a observarse las consecuencias graves en el ambiente no solo en una región, sino a nivel mundial. Un caso concreto es la formación de hoyos en la capa de ozono de la Tierra, lo que desembocó en la firma del Protocolo de Montréal (Naciones Unidas, 1987b) cuyo objetivo era frenar el uso de los clorofluorocarbonos, ${ }^{4}$ pues se descubrió que dañaban la capa de ozono.

\footnotetext{
3 El diclorodifeniltricloroetano, comúnmente conocido como DDT, es un compuesto químico cristalino incoloro, insípido y casi inodoro (un organoclorado). Es muy soluble en las grasas y en disolventes orgánicos, y prácticamente insoluble en agua. Originalmente se desarrolló como un insecticida, pero posteriormente se volvió abominable por sus negativos impactos ambientales y en la salud de las personas.
}

${ }^{4}$ Compuesto químico utilizado en sistemas de refrigeración y aerosoles. 
En esta década surge la teoría de los stakeholders o grupos de interés (Freeman, 1984), que reconoce a la empresa como un actor más en la sociedad y cuyas operaciones impactan, directa o indirectamente, a sus grupos de interés (stakeholders). Cabe señalar que, con base en esta teoría, más adelante algunos autores reconocerán el medio ambiente y el futuro mismo como otros dos stakeholders (Crowther y Aras, 2008).

Esta época también vio nacer la publicación del Informe Brundtland (Naciones Unidas, 1987a) y, con él, la primera definición «oficial» de desarrollo sostenible como «aquel desarrollo que permite cubrir las necesidades del presente sin comprometer la habilidad de futuras generaciones para cubrir sus necesidades» (Naciones Unidas, 1987a: 41). Desde entonces, esta definición ha sido la universalmente aceptada para definir el concepto de «sostenibilidad» y más adelante se desarrollaron modelos para poder concretarla.

Uno de los modelos precursores es el «Marco para el desarrollo estratégico sostenible» (A Framework for Strategic Sustainable Development, FSSD), que nace junto con la organización sueca The Natural Step en 1989. El FSSD es un modelo que plantea las condiciones básicas mínimas del sistema (Tierra) para la sostenibilidad estratégica de las actividades humanas; es decir, para un desarrollo estratégico sostenible. (Broman y Robèrt, 2017).

1990. A pesar del avance en teorías como la RSE o la de stakeholders y de una definición aceptada a nivel internacional de sostenibilidad, en los años noventa salen a la luz escándalos de trabajo infantil, explotación y condiciones laborales inapropiadas por empresas de ropa deportiva como Nike, Adidas y otras (Werner y Weiss, 2003). En la década de los noventa los temas de la no sostenibilidad («insosteniblidad») y cambio climático comienzan a aparecer en la prensa y en la agenda global.

En 1992 se celebra la Cumbre de la Tierra de Río de Janeiro, donde surge el Programa 21,5 que fue el primer esfuerzo para crear una agenda internacional enfocada en el medio ambiente y el desarrollo. Un mes después nace la Convención Marco de las Naciones Unidas sobre el Cambio Climático (UNFCCC, por sus siglas en inglés).

En 1995 se celebra la primera Conferencia de las Partes (Conference of the Parties, COP) en Berlín, que es la reunión anual que convoca el UNFCCC para reunir a

\footnotetext{
${ }^{5}$ Más conocido como Agenda 21 por su título original en inglés.
} 
jefes de Estado y líderes empresariales para tratar los temas de cambio climático y desarrollo sostenible, y busca acuerdos a nivel internacional así como desarrollar programas a nivel regional y nacional. Se comienzan a publicar trabajos que buscan integrar y equilibrar los tres factores que forman la sostenibilidad: social, ambiental y económico.

También en esta década surge en Estados Unidos e Inglaterra un interés en torno a la sostenibilidad y su relación con el sistema económico. En 1994, John Elkington (1997) plantea su ahora famoso modelo «triple resultado final», o triple bottom line, en el cual propone que las empresas integren en la contabilidad del negocio y en sus informes financieros sus actividades de responsabilidad social e impacto ambiental. Al final de la década, Hawken, Lovins y Lovins (1999) escriben el libro Natural Capitalism, en el que cuestionan el modelo económico prevaleciente y proponen que los sistemas ecológicos y los recursos naturales, al ser imprescindibles para la vida, deben considerarse y contabilizarse en un nuevo sistema económico y en nuevos modelos de negocios.

2000. Al inicio del siglo XXI nacen los ocho Objetivos de Desarrollo del Milenio (ODM), cuando los países miembros firman la Declaración del Milenio de la ONU (2000). Los Objetivos del Milenio se enfocaban en resolver problemáticas sociales, particularmente en erradicar la pobreza extrema, fijando una fecha límite: el año 2015. Es importante señalar que no solo los gobiernos, sino también los corporativos y el sector social emprendieron esfuerzos para el cumplimiento de los ODM, observándose buenos resultados, aunque no contundentes.

Los Objetivos del Milenio sentaron las bases para futuros programas más exhaustivos. En la primera década de siglo XXI, la problemática del cambio climático crece y se hace más clara, a la par que el avance en la tecnología y la automatización (Schwab, 2016) dejan de manifiesto la presencia inminente de la inteligencia artificial en nuestro presente, así como en el futuro (Hastings, 2018). En contraste, en esta década se suscitaron algunos de los fraudes corporativos más notorios como los de WorldCom, Enron, Freddie Mac, Lehman Brothers, AIG y otros.

2010. En la Asamblea General de la ONU de 2015 se creó la Agenda 2030 y con ella los 17 Objetivos de Desarrollo Sostenible (ODS) unos meses antes de la firma del Acuerdo de París, el cual se ha convertido en detonante en la discusión alrededor de la crisis climática y que busca involucrar a todos los sectores por igual a nivel internacional, con un llamado de urgencia a la acción. Los ODM planteados en 2000 y con fecha de expiración en 2015 se habían enfocado en problemáticas sociales. 
Debido al creciente número de desastres naturales a nivel internacional por alteraciones en el clima, la rápida pérdida de la biodiversidad, el deterioro ambiental y de la salud del ser humano, así como de la flora y fauna terrestres y marinas, se hacía necesario un planteamiento que abarcara también estos temas. Los ODS plantean un modelo que busca extender la agenda internacional de los ODM al ámbito medioambiental y de cooperación para combatir el cambio climático.

En 2018, el Panel Intergubernamental de Cambio Climático (IPCC, por sus siglas en inglés) publicó su último reporte en el que advierte que, de no tomar rápidamente acciones conjuntas gobiernos y empresas, el calentamiento global superará los $1.5^{\circ} \mathrm{C}$, lo cual dificultará la vida en la Tierra y, por ende, cualquier actividad productiva en el presente y en el futuro (IPCC, 2018).

\section{Análisis de los resultados}

\section{Ética empresarial}

El contexto histórico mencionado anteriormente en definitiva ha ido cambiando la forma de operar de las empresas. El sector empresarial ha tenido que modificar sus políticas de operación. Idealmente, las empresas vigilan más cuidadosamente su gobierno corporativo y sus códigos de ética, así como las relaciones con sus stakeholders. Los gobiernos, organismos certificadores y organismos multilaterales han actualizado sus regulaciones y legislaciones para alinearse a formas más éticas de operar, y adaptarse al entorno cambiante. De igual forma, las expectativas de la sociedad civil también se han transformado, ya que como consumidores nos hemos vuelto más conscientes de los impactos sociales y ambientales $y$, en consecuencia, somos más exigentes con los productos y servicios que adquirimos. Sin embargo, todavía existe una urgente necesidad de educar y responsabilizar tanto a la sociedad civil, como a los sectores privado, público y social del impacto social y ambiental que generamos como parte del ciclo producciónconsumo-desecho.

La ética, a pesar de las circunstancias de un mundo nuevo, no cambia (Savater, 2002), sin embargo, los valores y principios sí van cambiando, o evolucionando junto con el contexto. Como lo mencionó el filósofo Fernando Savater (2002) en una conferencia en Monterrey, Nuevo León: «La ética no es más que la reflexión sobre aquello que es valioso para la vida, lo que hace a la vida más digna de ser vivida por 
un ser humano». Podemos observar que actualmente los valores de equidad, no discriminación, cuidado al medio ambiente, entre otros, se van aunando al conjunto de valores personales y empresariales, porque se reconoce la importancia de cada uno de estos aspectos para una convivencia más armónica y relaciones más saludables tanto en lo personal, como en ámbitos profesionales. El respeto hacia la vida de otros seres no es un valor nuevo, pero cuando lo ponemos en el contexto empresarial y a la luz de hechos históricos que han incluso cobrado la vida de muchos seres humanos, seres vivos y la integridad de ecosistemas, empieza a dar forma a una cultura de valores empresariales que originan códigos de ética o códigos de conducta, como también se les denomina, y se vuelven indispensables para operar hoy en día en el mundo empresarial.

Como sociedad somos cada vez más exigentes con las empresas para que apoyen estos «nuevos», o actualizados valores. Las regulaciones, y los mismos consumidores les exigen mayor transparencia, que sus productos, servicios y programas reflejen una ética empresarial real y no superficial. Es decir, exigimos de las empresas una cultura organizacional con ética genuina y no cosmética e identificarla es relativamente sencillo en un mundo con tanta información al alcance de un teléfono celular o una tableta y una conexión a internet. Una empresa puede lanzar campañas publicitarias con «impacto social» efímero o hueco, o lo que se ha denominado «marketing social corporativo»6 (Licandro, 2016: 25), o campañas de «impacto ambiental» engañoso, o greenwashing7 (Delmas y Cuerel, 2011: 65), pero es relativamente fácil para el consumidor y demás stakeholders darse cuenta de que en realidad son campañas publicitarias cuya finalidad es el beneficio económico de la empresa.

La ética empresarial es un tema al que se le dedica cada vez más atención en la agenda de la alta dirección en el sector privado. Las empresas saben que, si no se alinean con los cumplimientos normativos, ya sean nacionales o internacionales, o si alguno de sus colaboradores tiene conductas poco o nada éticas, la organización puede verse sujeta a denuncias, sanciones, crisis de reputación y el rechazo

${ }^{6}$ «El marketing social corporativo es el conjunto de acciones mediante las cuales la empresa asigna recursos propios en favor de una causa social u organización no gubernamental, con el objetivo de obtener algún tipo de impacto sobre los activos intangibles que habitualmente gestiona la función de marketing: la marca, la reputación corporativa y la cartera de clientes» (Licandro, 2016: 25).

7 «Greenwashing es la intersección de dos comportamientos de la empresa: un pobre desempeño medioambiental y la comunicación positiva del desempeño ambiental» (Delmas y Cuerel, 2011: 65). 
de los mismos consumidores. Esto puede afectar sensiblemente las ventas y, por tanto, los resultados financieros, al grado de llevar a la organización al colapso, como en los casos antes mencionados de Nike, Adidas, WorldCom o Enron (Werner y Weiss, 2003).

Por lo anterior, hay una creciente preocupación de las empresas para conducirse de manera más ética. Muchas organizaciones se han apresurado, aunque erróneamente, en incorporar programas de responsabilidad social empresarial y/o modelos de sostenibilidad, temas que se tratarán más adelante. Sin embargo, cuando los valores y la ética del negocio no son claros, o no se les considera el eje rector de la operación, los programas de responsabilidad social o de sostenibilidad pueden funcionar temporalmente, pero acabarán por perder impulso o desmantelarse, ya que no tienen sustento en los valores de la empresa ni se alinean a la estrategia comercial, y esto se vuelve evidente para los stakeholders, comenzando por los mismos empleados.

Nuestros patrones de consumo y producción están guiados por nuestros valores y estos, a su vez, configuran nuestra propia ética. La ética, desde un punto de vista moral, es el código de conducta con el que guiamos nuestros actos a lo largo de la vida (Savater, 2002). Tácito o implícito, el líder de la organización está trasladando sus valores personales y su código de conducta a la empresa que dirige (Thulasivelu, 2012). Aunado a esto, las empresas están integradas por varios o muchos individuos, cada uno con su propio conjunto de valores; por eso es de vital importancia que las empresas implementen un código de ética con la suficiente claridad y difusión, que diseñen programas de capacitación apropiados y aplicables a lo largo y ancho de la organización. Drucker (1981) dice que «no hay ética empresarial», sino «ética en los negocios». Dicho de otra forma, e introduciendo los siguientes dos conceptos, no puede haber empresas sostenibles sin ética o sin prácticas de responsabilidad social. De igual forma, no puede haber empresas socialmente responsables sin que se conduzcan de forma ética.

\section{Responsabilidad social empresarial}

En los años ochenta, el término «ética empresarial» se convirtió en el término de «moda». Por ello, Drucker (1981) proponía mejor llamarla «ética chic», pues la consideraba más un evento mediático que un tema moral o filosófico. Un fenómeno similar sucede en la actualidad cuando se habla de «responsabilidad social empresarial». 
Si bien se confunde con filantropía, o se le considera una herramienta para coadyuvar al desahogo de problemas sociales, como la pobreza, el hambre, la desnutrición, la falta de educación, equidad de género, etcétera, también se ha convertido en un refugio, o placebo, para organizaciones que quieren hacer el bien o que saben que «tienen que hacer el bien a la comunidad», pero que no han pasado por un proceso de transformación profunda desde el interior de la organización, comenzando por los líderes y tomadores de decisiones, los valores empresariales y la cultura que permea toda la organización.

Desafortunadamente, una gran parte de la RSE se ha convertido, igual que la «ética chic» de Drucker, en un elemento mediático o de marketing para un gran número de empresas a nivel nacional e internacional. Existen aquellas que, a pesar de haber obtenido certificaciones ISO y otros distintivos, han mostrado prácticas irresponsables y escandalosas en lo social y en lo ambiental. Por lo mismo, se ha criticado la práctica de las certificaciones y distintivos como banal, debido a que ha dado pie a una industria lucrativa para organizaciones certificadoras o aquellas que otorgan distintivos de RSE (Muñoz-Martín, 2013). Cabe destacar que uno de los principios de la RSE es el "comportamiento ético», como lo indica una de las materias fundamentales de la norma ISO 26000.

Es digno de reconocer, como se mostró en el contexto histórico, que poco a poco las iniciativas, herramientas y regulaciones buscan mover la actividad productiva hacia una más responsable. En la actualidad, algunas empresas aspiran a convertirse, de forma genuina, en empresas socialmente responsables y así contribuir a un cambio social y medioambiental positivo. Sin embargo, cuando la RSE no es una iniciativa que surge de la convicción desde el corazón del negocio, sí se observa que es necesariamente impulsada por legislaciones, regulaciones, o bien, por la presión de la industria - los competidores que sí están implementando programas de RSE-, o por los mismos consumidores que demandan mayor responsabilidad de la empresa, así como mayor transparencia, tal y como se mencionó anteriormente. En cualquier instancia, resulta importante que las empresas se capaciten a todos los niveles con herramientas de RSE y empiecen a integrar prácticas de esta índole en todo lo largo y ancho de su operación.

Bowen hace hincapié en que los empresarios deben «aplicar políticas, tomar decisiones o seguir las líneas de acción que son deseables en términos de los objetivos y valores de nuestra sociedad» (Bowen, 1953: 6). Es decir, la empresa debe buscar una ganancia económica y también una forma de retribuir a la sociedad, debido 
a que forma parte y necesita de ella; por lo tanto, no puede operar aislada y de ahí su responsabilidad. Sin embargo, Bowen hizo énfasis en el empresario y no en la empresa, lo cual cambió rápidamente «con la "revolución" de la gestión de empresas y la creciente hostilidad pública tras experimentar problemas sociales» (Valor, 2005: 192).

Más adelante, en los años setenta, surge la teoría de la pirámide que propone que las empresas deben basar su actividad en cuatro factores: económico, legal, ético y filantrópico (Carroll, 1991). La teoría de Carroll afirma que el factor económico es la base de la pirámide y este no solo es necesario, sino imprescindible para la operación de la empresa. De igual forma, el segundo factor, el legal, también es un requisito para las empresas porque deben operar en el marco de la ley. El tercer factor es el ético, o hacer lo «correcto», y en esta época se le consideraba como «esperado» por parte de la empresa. El último es el factor filantrópico, que se consideraba «deseable» y como una característica que permitía a la empresa mostrar que era «responsable» (ver figura 1).

Figura 1. Pirámide de responsabilidad social corporativa según Carroll

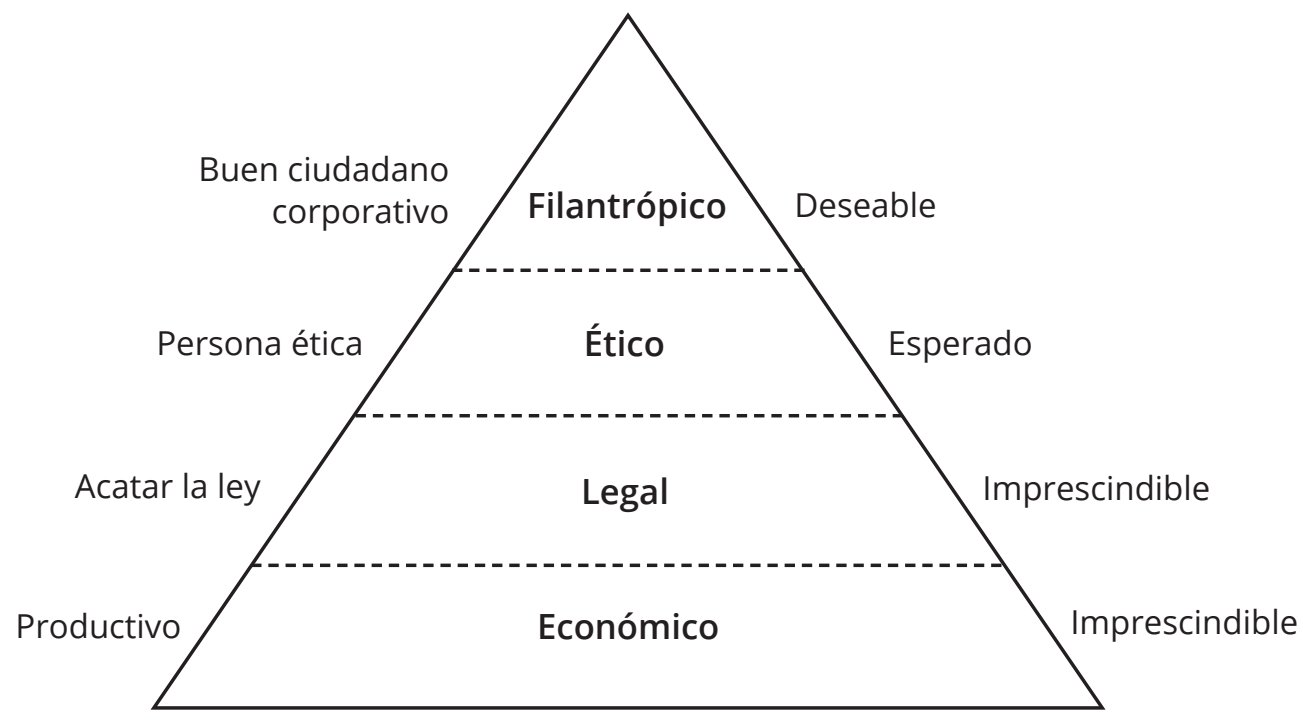

Fuente: Carroll (1991). 
Recordemos que la cultura de la filantropía empresarial en Estados Unidos lleva ya más de un siglo por lo que, en la época de la pirámide de Carroll, el factor filantrópico de la «responsabilidad empresarial» no resulta tan ajeno. Cabe destacar que la RSE no es solo contar con programas de filantropía, donaciones o programas comunitarios. En países de Latinoamérica, la RSE se ha enfocado mucho en la filantropía (Weyzig, 2006) y en programas sociales, desdeñando la responsabilidad de la empresa por su impacto en el medioambiente o su relación e impacto entre grupos de interés más cercanos, por ejemplo, sus empleados o clientes. El término de «stakeholders», o partes interesadas, surgió con la teoría de los stakeholders (Freeman, 1984), que explica que las partes interesadas son «cualquier grupo o individuo que pueda afectar o verse afectado por el logro de los objetivos de la organización». Aunque la filantropía y los programas sociales son actividades importantes, que sí forman parte de la RSE, no suplen las responsabilidades de la empresa con sus demás stakeholders: empleados, proveedores, clientes, accionistas, comunidad, medio ambiente, gobierno y reguladores, competidores, etcétera. Incluso hay algunos autores que consideran al futuro un stakeholder, entendiendo por futuro el propio y el de las siguientes generaciones. La RSE y el concepto de stakeholders se complementan y refuerzan entre sí (Valor, 2005).

Un aspecto de la RSE es que tiende a verse como una herramienta que se lleva a cabo solo cuando la situación económica de la empresa es favorable o cuando se percibe como necesaria (Vives, 2006). Otro aspecto de la RSE, que la hace compleja, es que es voluntaria; es decir, su carácter es moral y no legal. La RSE trata de ir más allá porque pretende mejorar las condiciones de la empresa y su entorno; por lo mismo, su aceptación e implementación representa un reto. En el momento que la RSE se legisle, la responsabilidad social perdería su esencia y objeto, pues las empresas tendrían que cumplir por normativa, no por convicción. Derivado de lo anterior, la RSE se ha adoptado como un sistema de gestión.

Algunos de los beneficios de la RSE es que ha incrementado la competencia de las empresas que la implementan, por ello, se vuelven lugares de trabajo deseables y atraen empleados talentosos, aumenta la tasa de retención de los colaboradores y la tasa de productividad en empresas con programas de RSE también crece. De igual forma, puede mostrar más altas tasas de fidelización de clientes y mayor confianza en los mercados. Al igual que sucede con el tema de la ética empresarial, si la RSE se implementa de manera artificial o superficial, se vuelve notorio eventualmente. Como dice Muñoz-Martín (2013: 78), citando a Davis (1990), «la sociedad concede legitimidad y poder a la empresa. En el largo plazo aquellos 
que no usan ese poder de una manera que la sociedad considere responsable, tienden a perderlo». Es decir, la «licencia social» que la sociedad ha otorgado implícitamente a la empresa al comenzar sus operaciones, se le retira una vez que la sociedad misma, o varios stakeholders, detectan iniciativas de RSE sin sustento o falsas.

Para poder extender la aplicación de los programas o sistemas de gestión de RSE y que tengan un impacto completo, es decir, considerando las implicaciones de impactos ambientales y no solo los impactos sociales a nivel local, es importante contar con un marco de referencia de sostenibilidad integral.

\section{Sostenibilidad empresarial}

Se ha discutido cómo la ética empresarial es la base para el desarrollo de genuinas prácticas de RSE. A su vez, esta ha dado una nueva luz a las empresas y les ha permitido ver más allá de la rentabilidad financiera para considerar su impacto social y el medioambiental. Solo por mencionar un ejemplo de normas o certificaciones, entre las muchas que existen, la norma ISO 26000 considera el medio ambiente en el abanico de la responsabilidad social de la empresa, específicamente en su quinta materia fundamental de las siete que la forman, pero queda muy limitada, porque no explica ni da guía sobre el papel de la empresa y su impacto en un contexto mayor. Por otra parte, el estándar del Global Reporting Initiative (GRI), aunque proporciona un mayor detalle en sus parámetros para evaluar a las empresas, sigue quedando en un nivel de evaluación y análisis sin que explique el papel de la empresa en el contexto global y su contribución en el desarrollo sostenible. Adicionalmente, los especialistas en el tema coinciden en dos cosas: a) que es común encontrar incongruencias en la información de las empresas de un año al siguiente, y b) que la tendencia es que se le utiliza como una herramienta publicitaria en un espíritu de autocomplacencia.

La empresa desempeña un papel importante no solo en una localidad, sino en un contexto global, especialmente en el mundo por demás globalizado e interconectado donde dependemos de materiales y mano de obra provenientes de los rincones más alejados, sin que necesariamente lo sepa el consumidor final. Adicionalmente, el objetivo de conseguir una certificación, distintivo o estándar de RSE o Sostenibilidad puede sesgar el propósito de las empresas, al buscar estos certificados únicamente con fines publicitarios o, en el mejor de los casos, por cumplimiento normativo. 
El triple-bottom line de Elkington (1997) consideró tres pilares: económico, social y medioambiental para incluir en la contabilidad y reportes financieros de las empresas. Fue un buen esfuerzo proponer una herramienta que llevara al mundo empresarial la conciencia de considerar más que solo los datos duros de lo que ya se contabilizaba materialmente. Sin embargo, el mismo Elkington «retiró» su triplebottom line como concepto en la gestión empresarial (Elkington, 2018), pues reconocía que había quedado limitado como otro sistema contable dentro de la comunidad empresarial y que no había impulsado lo que pretendió su autor al proponerlo en los años noventa: un cambio transformador al interior de la empresa.

El modelo sueco denominado "Marco estratégico para el desarrollo sostenible» (Framework for Strategic Sustainable Development o FSSD por sus siglas en inglés) que proponen Broman y Robèrt (2017), pone en contexto a la empresa, desde el pensamiento sistémico, como un subsistema de la sociedad, y a la sociedad como un subsistema del medio ambiente o Tierra, del que los dos primeros son dependientes. Este marco, desarrollado y revisado con bases científicas, establece originalmente cuatro condiciones sistémicas básicas para que el sistema Tierra funcione de manera apropiada y continúe soportando a los otros subsistemas que dependen de él. El FSSD establece también un marco estratégico de cinco etapas para mover cualquier proyecto u organización hacia operaciones más sostenibles. Este modelo o marco de referencia ayuda a concretar la definición del Informe Brundtland (Naciones Unidas, 1987a) y plantea una metodología que ayuda a avanzar de manera estratégica para crear una empresa sostenible, eliminando así la percepción de que el camino deba ser oneroso o abrumador.

En la actualidad, la sostenibilidad ha tomado tal relevancia que ya forma parte de la agenda empresarial y, en los últimos años, se ha convertido en prioridad a nivel global. Tanto es así, que incluso el Business Roundtable está redefiniendo el propósito de la existencia de las corporaciones con el artículo que publicó en agosto de 2019, en el que reconoce que es importante para las empresas darle mayor peso a los stakeholders sobre los shareholders (o accionistas), e incluso ya empiezan a considerar la importancia de «proteger el medio ambiente adoptando prácticas sostenibles en todos nuestros negocios» (Business Roundtable, 2019).

En México, de acuerdo con la encuesta Perspectivas de Alta Dirección de la consultora KPMG (2019), la preocupación número uno de los 993 empresarios encuestados es la corrupción. Aquí la ética se vuelve un factor de particular relevancia. Y en la última encuesta de Desarrollo Sostenible de la misma consultora (2018), el 98\% de los empresarios encuestados considera la sostenibilidad «crítica y relevante para el 
futuro de su empresa». Las iniciativas de responsabilidad social son cada vez más importantes y cobran cada vez mayor popularidad, sin embargo, se reconoce que el tema de sostenibilidad engloba tanto a la RSE, como a la ética empresarial.

Llegados a este punto, es de suma importancia aclarar la diferencia entre sustentabilidad y sostenibilidad, pues existe mucha confusión, así como diversas e incorrectas interpretaciones. El primero, sustentabilidad, es un término que suele usarse en muchas partes de Latinoamérica, aunque de manera errónea. El verbo sustentar ${ }^{8}$ existe y es correcto, sin embargo, sustentabilidad es una palabra que no existe en el idioma español, sino que es un anglicismo de la palabra «sustainability». Por otra parte, "sostenibilidad» es la traducción correcta al español y, de acuerdo con el Diccionario de la Real Academia Española (DRAE, s/f), se define lingüísticamente como: "cualidad de sostenible». Asimismo, sostenible es un adjetivo y se define como aquello «especialmente en ecología y economía, que se puede mantener durante largo tiempo sin agotar los recursos o causar grave daño al medio ambiente».

Dicho lo anterior, el concepto de sostenibilidad es integral porque abarca: a) la ética empresarial, la cual dicta la forma «correcta» de operar respetando a los stakeholders, siendo responsable y evitando riesgos innecesarios para la empresa; b) la gestión y responsabilidad de la empresa por sus actividades e impactos sociales y ambientales, ante todos sus stakeholders, es decir, la responsabilidad social empresarial; y c) plantea un contexto en el que dentro del sistema complejo «Tierra» existen otros dos subsistemas dependientes: el sistema «sociedad» y su subsistema «económico», es decir, las empresas y gobiernos, así como sus transacciones. Por lo tanto, la Tierra es también un stakeholder del que dependen los otros subsistemas, o stakeholders, y sin el cual no pueden existir. Un modelo o marco de sostenibilidad difícilmente podrá mantenerse a lo largo del tiempo, o ser «sostenible», si no existe una sólida ética empresarial, como ya se mencionaba, que permita a la empresa cuestionar y gestionar su papel como parte de la sociedad y como responsable ante ella, así como su dependencia del medio ambiente. Lo anterior es lo que plantea y hace relevante al modelo FSSD como un marco único de estrategia para la sostenibilidad.

\footnotetext{
8 La Real Academia Española define sustentar como: proveer a alguien del alimento necesario; conservar algo en su ser o estado; sostener algo para que no caiga o se tuerza; defender o sostener determinada opinión.
} 


\section{Recomendaciones}

\section{Recomendaciones sobre ética empresarial}

Si una organización está interesada en temas de responsabilidad social y sostenibilidad, es conveniente que articule las bases en las que se establece y que estas sean claras y entendibles. Lo anterior es válido independientemente de si la empresa es de reciente creación o si ha estado establecida durante años. Hacer este ejercicio le dará mayor formalidad y profesionalismo a la empresa comprometida con ser una organización más sostenible y socialmente responsable. Los pilares de la empresa, articulados en un código de ética, idealmente confirmarán lo que la compañía representa o quiere representar; le dará un propósito a toda la organización y servirá como directriz tanto en tiempos de éxito, como de crisis. Visto desde otro punto de vista, también es una declaración sobre el compromiso de la organización ante todos sus stakeholders.

Es importante que, para convertirse en una empresa socialmente responsable de manera exitosa, la organización cuente primero con una sólida ética empresarial basada en principios y valores, es decir, que sea responsable de origen. Esto facilitará que al integrar políticas y programas de RSE, estos sean recibidos con mayor facilidad por los colaboradores o empleados, o stakeholders internos, «fundamental[es] para la gestión de la empresa» (Kliksberg, 2000: 21) y los primeros en recibir las iniciativas empresariales y transmitirlas o comunicarlas a los stakeholders externos.

De igual forma, es muy importante que las empresas cuenten con un sistema de gestión apropiado en su búsqueda de talento para contratar a los colaboradores cuyos valores se alineen a los de la empresa, pues esto facilitará la consecución del código de ética que ha diseñado la organización y favorecerá su productividad. Por ello, es vital contar con políticas de contratación, una gestión de recursos humanos apropiados, un buen gobierno corporativo pero, sobre todo, con un código de ética articulado que dicte con claridad cómo deben dirigirse los miembros de la organización y que este sea igualmente aplicable para los altos directivos y para los niveles más básicos (Crowther y Aras, 2008).

Dos prácticas cruciales para las empresas son los programas de inducción de personal sobre el código de ética y contar con programas de capacitación continua sobre este, pues la repetición de las directrices con las que se conduce una organización resulta clave para prevenir riesgos por conductas inadecuadas o no éticas (Thulasivelu, 2012). 


\section{Recomendaciones sobre responsabilidad social}

Es indispensable en la actualidad, y en el futuro lo será también, que las empresas eleven sus estándares de operación a unos más éticos y responsables, pues se ha vuelto la norma y la exigencia mínima en el mercado; estar dentro del marco jurídico ya no es suficiente. En una sociedad tan informada e interconectada, las empresas también tienen que tomar la iniciativa de informarse sobre lo que significa ser socialmente responsable y sobre cómo aplicar y gestionar prácticas de RSE en las operaciones diarias.

Las empresas tienen una posición privilegiada porque pueden mostrar liderazgo y dictar el rumbo. Una forma de hacerlo es actualizar sus códigos de ética, e incluso sus valores empresariales, si es necesario, para que estén alineados a una cultura conducente a la responsabilidad social. Otra forma es integrarla estratégicamente en las operaciones de la empresa de manera que tenga sentido y se alinee con sus objetivos comerciales de manera natural. De lo contrario, como ya se ha mencionado, sus stakeholders podrían percibir las iniciativas y programas como efímeros, faltos de sustento o simplemente falsos.

Cabe destacar que la responsabilidad social no es exclusiva del sector privado, sino que se extiende a todos: sector público, sector social y a la sociedad civil misma. Esto es, la responsabilidad social es de todos, tanto de organizaciones, como de los diversos sectores, pero también de todos como individuos, puesto que formamos parte de uno o varios sectores a la vez y es también con base en nuestros hábitos de consumo individuales que las empresas desarrollan productos, servicios y mercados para satisfacerlos. Sin embargo, las empresas, como agente productor y con gran alcance e influencia, tienen la responsabilidad de «educar» a sus clientes y consumidores finales y así promover productos, servicios, políticas y procedimientos responsables con la sociedad y el medioambiente. Por lo anterior, es importante que se considere a los distintos stakeholders en los procesos de la organización. Con ello, las empresas tienen la oportunidad de convertirse en líderes de cambio, fidelizando a sus actuales clientes y atrayendo a nuevos, que buscan favorecer a las organizaciones con estándares más altos de ética y responsabilidad.

Mientras se profundiza el conocimiento de la RSE tanto dentro, como fuera de las organizaciones $y$, a pesar de las críticas sobre si el mercado de las certificaciones 0 distintivos es banal y lucrativo, las certificaciones continúan siendo un parámetro de mejora, las ayudan a tener mayor proyección, les dan acceso a redes de negocios, 
cámaras de comercio o cámaras industriales y, en general, siguen «impulsando» el mercado y las empresas hacia estándares más altos.

En materia de responsabilidad social, un estandarte de buenas prácticas y una guía muy útil para las empresas es la Norma ISO 26000. Sin embargo, como ya se mencionó, es limitada. Por lo tanto, es recomendable que cada organización no solo cumpla con los requisitos de la ISO y otras normas, distintivos y certificaciones, sino que exceda tales estándares, ya que con ello asegurará sus operaciones ante futuras regulaciones y exigencias del mercado. Para lograrlo, lo principal y más importante es que exista una transformación interna así como que la organización tenga la disposición de ser más responsable ante la sociedad y más sostenible de manera integral. En virtud de ello, resultan muy útiles algunos marcos de sostenibilidad que se mencionan en el siguiente apartado, dado que hacen las veces de brújula y marcan el rumbo.

\section{Recomendaciones sobre sostenibilidad}

Como se ha visto a lo largo del presente artículo, puede decirse que la ética es la base para organizaciones interesadas en tener, o desarrollar, un plan estratégico de sostenibilidad. Si una empresa quiere ser sostenible, debe ser muy cuidadosa en el tipo de cultura que priva en la organización. Idealmente, los directivos son quienes debieran generar una cultura con base en principios éticos. Adicionalmente, también son los líderes de una organización quienes deben estar convencidos de los beneficios de llevar a su empresa hacia prácticas más sostenibles, así como de conocer los riesgos de no tomar acciones para prevenirlos. Igualmente importante es que el o los líderes tengan el compromiso de llevar a cabo un cambio en esta dirección.

Toda organización tiene problemáticas y retos propios y, por supuesto, no puede atender todos los temas socioambientales globales, sino los que le atañen o afectan directamente. Con una genuina disposición de tener un impacto positivo, muchas empresas cometen el error de buscar ansiosamente herramientas de gestión y seleccionar las «de moda», las más conocidas o las que estiman más útiles, pero no se aseguran antes de: a) tener claridad en el concepto de sostenibilidad y lo que esto significa para su organización y, por ende, b) comprender si los objetivos de sostenibilidad o de RSE que se han planteado contribuirán a la estrategia comercial y llevarán a su organización, y a la sociedad en general, hacia el desarrollo sostenible. Esta falta de estrategia, en lugar de acercarlos a convertirse en empresas más 
sostenibles, los aleja de ello, puesto que deja procesos inconclusos, equipos frustrados y gastos no inteligentes e innecesarios.

La forma de evitar estos errores es informarse con expertos sobre las mejores estrategias, los modelos de sostenibilidad y la implementación indicada para su organización. Es muy importante que un plan integral de sostenibilidad considere el impacto social y ambiental de la empresa, así como su desempeño financiero, siempre en un contexto de largo plazo.

Conjuntamente con informarse y allegarse de expertos, el líder de la organización también debe comunicar a su equipo, a toda la organización y a sus stakeholders sobre sus compromisos de dirigirse hacia prácticas más sostenibles. Es fundamental tener un programa de capacitación interno (incluso externo si se considera necesario), para el éxito del plan de sostenibilidad integral y las estrategias que de este se deriven. Es crucial transmitir a los colaboradores tales estrategias o, en su caso, la nueva dirección que tome la organización, pues este mensaje lleva la impronta del compromiso organizacional y de los ejecutivos. Poder transmitir este compromiso es clave en el proceso y, sobre todo, está ligado al éxito del plan y de su implementación. Dos vías para lograrlo son elegir los canales adecuados de comunicación interna y brindar la capacitación apropiada para la organización.

Conviene contar con un modelo que dé guía y enmarque las estrategias y procesos y que ayude a identificar herramientas de monitoreo, evaluación y medición. Un marco de trabajo que da los parámetros de cómo podría verse la sostenibilidad a nivel de empresa e incluso global, es el marco sueco FSSD (Broman y Robèrt, 2017). Este modelo explica los parámetros de lo qué es y lo qué no es sostenible; plantea los principios y las condiciones sistémicas básicas para crear organizaciones más sostenibles. Al seguir este modelo, la empresa interesada tendrá entonces la guía de cómo generar acciones de alto impacto para la sociedad, beneficiando además a cada uno de sus stakeholders. EI FSSD ayuda a las organizaciones a lo siguiente: a) identificar sus recursos, necesidades, retos, riesgos y oportunidades; b) diseñar un plan de acción con sus distintas fases y elegir las herramientas que se identifiquen como las más convenientes para mediar y c) evaluar el progreso del plan de acción o programa integral de sostenibilidad de la organización. 


\section{Conclusiones}

Este artículo ha planteado cómo la ética, o su ausencia, nos ha llevado a la actual crisis ambiental y social. Se ha mostrado cómo ha evolucionado la ética empresarial desde hace casi un siglo, comenzando por fijar salarios mínimos y condiciones laborales apropiadas, pasando por temas de derechos civiles, justicia social y equidad, hasta llegar a incluir el cambio climático y su impacto medioambiental como parte de la responsabilidad de empresas y organizaciones ante la sociedad. Es decir, se ha mostrado cómo la ética empresarial ha ido ampliando su espectro y es la raíz del desarrollo sostenible y la base para la sostenibilidad en el mundo de los negocios. Finalmente, se plantean varias recomendaciones para que las empresas puedan acercarse más a ser sostenibles y alinearse con un presente y un futuro que demanda mayor responsabilidad y compromiso hacia la sostenibilidad.

La ética es lo que soporta tanto las prácticas de responsabilidad social, como los marcos de trabajo de sostenibilidad integral. Es difícil considerar que una empresa sea sostenible si no se conduce con prácticas y principios que buscan construir un mundo más justo para todos. Las empresas que cuenten con un programa integral de sostenibilidad, tienen la brújula que las conduce a operaciones más eficientes, mejor desempeño financiero, mejor ambiente de trabajo, equipos de trabajo más comprometidos, base creciente de clientes satisfechos e incremento en la fidelización. Además, lo antes señalado aporta al desarrollo propio de la organización y contribuye también al desarrollo sostenible de la sociedad en general. Sin embargo, para llegar a estos resultados, que sí son reales para muchas organizaciones alrededor del mundo, es necesario un proceso de transformación profunda de la organización.

Stephen Guerin, científico en sistemas complejos, mencionó en una conferencia que «cuando se cambia el paradigma (o modelo mental), el [nuevo] paradigma "fija" la ética del sistema» (Guerin, 2019). Por lo tanto, el cambio climático o crisis climática, está forzando a un cambio de paradigma, a un nuevo modelo mental en donde la nueva ética es no permitir que ni empresas, ni gobiernos, ni sociedades sigan perpetrando abusos e impactos negativos sobre los sistemas naturales que sostienen nuestra vida como especie en la Tierra, y de la vida de todas las otras especies y ecosistemas que tejen el complejo y delicado balance en el planeta.

Por lo tanto, los nuevos modelos de negocios deben nacer y convertirse en la nueva normalidad. Al observar que las condiciones externas cambian y los modelos mentales también, la respuesta inmediata de los líderes empresariales más visionarios 
y exitosos es informarse, educarse y consultar con expertos en el tema para efectuar los cambios y adecuaciones necesarias para que su organización pueda adaptarse, sea competitiva y se adelante al futuro y a los futuros mercados.

Considerando lo expuesto en el presente artículo y con base en el trabajo publicado tanto en Latinoamérica, como en América del Norte (anglosajona) y partes de Europa, la autora ha desarrollado una metodología de cinco etapas para apoyar a las empresas comprometidas en avanzar a procesos y sistemas que ayudan a las organizaciones a ser más sostenibles. Las cinco etapas son: 1) claridad, 2) visión, 3) compromiso, 4) evaluación y 5) despegue. Estas etapas forman parte de las asesorías, clases y tutoría que la autora está impartiendo desde febrero de 2020, en forma online (en línea), para tomadores de decisión en diversas organizaciones.

La primera etapa, claridad, consiste en tener una comprensión del concepto de sostenibilidad y lo que significa para la organización. La etapa de visión permite que la organización pueda hacer una planificación en el largo plazo, con base en parámetros que favorecen la sostenibilidad. En el compromiso, la tercera etapa, se diseñan los programas «a la medida» de la organización para lograr los objetivos e implementación de las estrategias de sostenibilidad. La cuarta fase es la de evaluación, en la cual, por medio de indicadores puede medirse el avance del programa o proyecto. Y la fase final es la etapa de implementación de los programas, en la que se ponen en práctica en la empresa. 


\section{Referencias bibliográficas}

Bowen, H. (1953). Social Responsibilities of Businessmen. Nueva York: Harper.

Broman, G. I. y Robèrt, K.H. (2017). «A framework for strategic sustainable development». Journal of Cleaner Production. Vol. 140, parte 1: 17-31.

Business Roundtable (2019). «Corporate Governance. Business Roundtable Redefines the Purpose of a Corporation to Promote "An Economy That Serves All Americans"». Business Roundtable. https://www.businessroundtable.org/ business-roundtable-redefines-the-purpose-of-a-corporation-to-promotean-economy-that-serves-all-americans

Carroll, A.B. (1991). «The Pyramid of Corporate Social Responsibility: Toward the Moral Management of Organizational Stakeholders». Business Horizons, 34 (4): 39-48. Carson, R (1962). Silent Spring. Nueva York: Houghton Mifflin Company

Convención Marco de las Naciones Unidas sobre Cambio Climático (1992). http:// unfccc.int/resource/docs/convkp/convsp.pdf

Crowther. D. y Aras, G. (2008). Corporate Social Responsibility. Boulder, CO: Ventus Publishing.

Davis, K. (1990). «Five propositions for Social Responsibility», citado en Hoffman, W. y Mills, J. (2006). Business Ethics. New Jersey: Prentice Hall.

Delmas, M. y Cuerel B., V. (2011). «The Drivers of Greenwashing», Management Review, vol. 54, núm. 1: 64-87.

Diccionario de la Real Academia Española, DRAE (s/f). https://dle.rae.es/

Dickie, W. (1995). Clair C. Patterson, Who Established Earth's Age, Is Dead at 73. The

New York Times. https://www.nytimes.com/1995/12/08/us/clair-c-patterson-whoestablished-earth-s-age-is-dead-at-73.html

Drucker, P. F. (1981). «What is "Business Ethics"?». The Public Interest, 63: 18-36.

Elkington, J. (1997). Cannibals with forks: the triple bottom line of $21^{\text {st }}$ century business. Oxford: Capstone.

Elkington, J. (2018). «25 Years Ago I Coined the Phrase “Triple Bottom Line." Here's Why It's Time to Rethink It». Harvard Business Review. https://hbr.org/2018/06/25years-ago-i-coined-the-phrase-triple-bottom-line-heres-why-im-giving-up-on-it

Federal Register: 41 Fed. Reg. 52977 (3 de diciembre de 1976).

Federal Register: 42 Fed. Reg. 44801 (7 de septiembre de 1977). Shareholder and Corporate Governance Issues. Library of Congress.

Freeman, R. E. (1984). Strategic Management: A Stakeholder Approach. Mershfield, MA: Pitman Publishing. 
Global Reporting Initiative, GRI. https://www.globalreporting.org/Pages/default.aspx Guerin, S. (2019). «Complex Adaptive Systems» (conferencia), en The Complex Systems Science Gathering. Estocolmo, Suecia.

Hastings, M. (2018). «Trabajar de forma más inteligente y sostenible. Aguardando una nueva ola» (junta ejecutiva y conferencia), México: KPMG.

Hawken, P., Lovins, A. y Lovins, L.H. (1999). Natural Capitalism, Creating the Next Industrial Revolution. Nueva York: Little, Brown and Company.

Intergovernmental Panel for Climate Change, IPCC (2018). Global Warming of $1.5^{\circ} \mathrm{C}$.

An IPCC Special Report on the impacts of global warming of $1.5^{\circ} \mathrm{C}$ above pre-industrial levels and related global greenhouse gas emission pathways, in the context of strengthening the global response to the threat of climate change, sustainable development, and efforts to eradicate poverty. https://www.ipcc.ch/sr15/

ISO 26000 Responsabilidad Social (2010). ISO 26000 Visión general de proyecto. https://www.iso.org/files/live/sites/isoorg/files/archive/pdf/en/iso_26000_ project_overview-es.pdf

Kaufman, B. E. (2012). Wage Theory, New Deal Labor Policy, and the Great Depression: Were Government and Unions to Blame? ILR Review, 65 (3): 501-532. https://doi. org/10.1177/001979391206500302

Kliksberg, B. (2000). Capital social y cultura. Claves olvidadas del desarrollo. Documento de divulgación 7. Buenos Aires: Banco Interamericano de Desarrollo e Instituto para la Integración de América Latina y el Caribe - INTAL.

Kolk, A. (2016). «The social responsibility of international business: From ethics and the environment to CSR and sustainable development». Journal of World Business. Elsevier, vol. 51: 23-34.

KPMG (2018). How to report on the SDGs? What good looks like and why it matters. KPMG. https://home.kpmg/xx/en/home/insights/2018/02/how-to-report-on-thesdgs.html

KPMG (2018). Encuesta de Desarrollo Sostenible en México. KPMG. https://home. kpmg/mx/es/home/tendencias/2018/02/encuesta-desarrollo-sostenible-enmexico-2018.html

KPMG (2019). «KPMG presenta "Perspectivas de la Alta Dirección en México 2019. Esfuerzos colaborativos para el crecimiento"». KPMG. https://home.kpmg/mx/ es/home/sala-de-prensa/press-releases/2019/02/kpmg-presenta-perspectivasde-la-alta-direccion-en-mexico-2019.html

Licandro, O. (2016). La responsabilidad social empresaria. Reflexiones, investigaciones y casos. Montevideo: Editorial Grupo Magro. 
Lozano, J. M. (2004). La responsabilidad social corporativa: una nueva forma de gestión empresarial, México: ESADE.

Muñoz-Martín, J. (2013). «Ética empresarial, responsabilidad social corporativa (RSC) y creación de valor compartido (CVC)». Journal Globalization, Competitiveness \& Governability, 7(3): 76-88. https://doi.org/10.3232/GCG.2013.V7.N3.05

Naciones Unidas, ONU (1945). Carta de las Naciones Unidas. https://www.un.org/es/ sections/history-united-nations-charter/1945-san-francisco-conference/index. html.

Naciones Unidas, World Commission on Environment and Development (1987a). Our Common Future. The Brundtland Report. Nueva York: Oxford University Press. Naciones Unidas, Programa de las Naciones Unidas para el Medio Ambiente, PNUMA (1987b). Protocolo de Montreal. https://www.unenvironment.org/ ozonaction/who-we-are/about-montreal-protocol .

Naciones Unidas, Programa 21, Cumbre para la Tierra de 1992, Río de Janeiro.

Naciones Unidas, ONU (2000). Objetivos de Desarrollo del Milenio. https://www. un.org/millenniumgoals/bkgd.shtml

Naciones Unidas, ONU (2015). Objetivos de Desarrollo Sostenible. https://www. un.org/sustainabledevelopment/es/2015/09/la-asamblea-general-adopta-laagenda-2030-para-el-desarrollo-sostenible/

Robert, K.H.; Holmberg, J.; Daly, H. y Hawken, P. (1997). «A compass for sustainable Development». International Journal of Sustainable Development and World Ecology, 4 (2): 79-92.

Robèrt, K.H. y Broman, G. (2017). «A framework for strategic sustainable development». Journal of Cleaner Production, vol. 140, parte 1: 17-31.

Savater, F. (2002). «La ética en el mundo de hoy» (conferencia). Monterrey, Nuevo León: Tecnológico de Monterrey.

Savater, F. (2014). Ética para la Empresa. Barcelona: Conecta.

Schwab, K. (2016). La cuarta revolución industrial. World Economic Forum.

Thulasivelu, K. (2012). Business Ethics, Corporate Social Responsibility and Governance. India: Sasurie College of Engineering, Department of Management Sciences.

Valor, C. (2005). «Corporate Social Responsibility and Corporate Citizenship: towards Corporate Accountability». Business and Society Review, 110 (2): 191-212.

Vives, A. (2006). «Social and environmental responsibility in small and medium enterprises in Latin America». The Journal of Corporate Citizenship, 21: 85-94.

Werner, K y Weiss, H. (2003). El libro negro de las marcas: el lado oscuro de las empresas globales ( $3^{a}$. ed.). Buenos Aires, Editorial Sudamericana. 
Weyzig, F. (2006). «Local and global dimensions of corporate social responsibility in Mexico». The Journal of Corporate Citizenship, vol. 24: 39-50.

\section{Sesiones y asesorías directas}

G. Broman y K.H. Robèrt (2005). Programa de posgrado Master's for Strategic Leadership towards Sustainability. Blekinge Tekniska Högskola, Karlskrona, Suecia.

Robèrt, K.H. (2005). Karlskrona, Suecia.

\section{Obras consultadas}

Carroll, A. B. (2000). «The Four Faces of Corporate Citizenship». En Richardson, J. E. (ed.). Business Ethics 00/01. Nueva York: Dushkin/McGraw-Hill: 187-191.

Cedillo, C.; Garcia-French, M.; Hordijk, R. y Lana Olup, K.N. (2012). «Four Case Studies on Corporate Social Responsibility: Do Conflicts Affect a Company's Corporate Social Responsibility Policy?». Utrecht Law Review, vol. 8, núm. 3.

Díaz, R. (2017). «La relación entre desempeño financiero y responsabilidad social empresarial (un estudio de 63 empresas emisoras de la Bolsa Mexicana de Valores)». The Anáhuac Journal, vol. 17, núm. 1.

Duque, Y., Cardona, M. y Rendón, J. (2013). «Responsabilidad Social Empresarial: Teorías, índices, estándares y certificaciones». Cuadernos de Administración, vol. 29, núm. 50: 196-206.

Gardiner, R. (2018). «Ethical Responsibility - An Arendtian Turn». Business Ethics Quarterly 28: 1.

GlobeScan \& SustainAbility (2017). Evaluating Progress toward the Sustainable Development Goals. https://globescan.com/evaluating-progress-towards-the-sdgs/

GlobeScan (2018). The 2018 GlobeScan-SustainAbility Leaders Survey. https://globescan.com/2018-sustainability-leaders-report/

Goel, M. y Ramanathan, P. (2014). «Business Ethics and Corporate Social Responsibility. Is there a dividing line?». ScienceDirect. Procedia Economics and Finance. Elsevier, vol. 11: 49-59.

González, A., (2018). «Re-thinking social responsibility and business ethics. A review of the thinking of Gilles Lipovetsky». Gestión y estrategia, núm. 53. 
Haces, J.A. (2015) Motivaciones de la responsabilidad social corporativa en Latinoamérica. The Anahuac Journal, vol. 15, núm. 2: 11.

Nizam, E.; Ng, A.; Dewandarub, G.; Nagayevc, R. y Nkoba, M.A. (2019). The impact of social and environmental sustainability on financial performance: A global analysis of the banking sector. Journal of Multinational Financial Management, Elsevier. Volume 49(C): 35-53. 


\section{Sobre la autora}

Itzel Orozco tiene un MBA en Business Administration, con especialidad en Empresa Sostenible por la Universidad de Oregon; maestría en Estrategia y Sostenibilidad, por el Blekinge Institue of Technology de Suecia, y economista por la Universidad Tecnológica de México. Es especialista en sostenibilidad, conferencista y catedrática, con más de 20 años de experiencia profesional en más de diez países como consultora. Cofundadora de la US Green Chamber of Commerce, Capítulo México. Ha diseñado cursos y talleres para el sector empresarial y la academia para más de mil alumnos y es coautora del libro Modelando el emprendimiento social en México, editado por LID y el IPADE Business School.

hola@itzelorozco.com 